\title{
EVOLUÇÃO FUNCIONAL DA REPARAÇÃO DO MENISCO POR IMPLANTE ABSORVÍVEL
}

\author{
FUNCTIONAL EVOLUTION OF MENISCAL REPAIR \\ USING ABSORBABLE IMPLANTS
}

Waldo Lino Júnior

\section{RESUMO}

Objetivo: Avaliar a evolução funcional dos joelhos submetidos à reparação da ruptura longitudinal do menisco com o implante absorvível flecha (arrow). Métodos: Entre junho de 1997 e fevereiro de 2001, 23 pacientes com idade média de 26 anos foram avaliados. O seguimento médio foi de quatro meses (45-96). Realizaram-se 19 reparações mediais e quatro laterais. Os pacientes foram avaliados no pré e no pós-operatório quanto à função, de acordo com a escala de Lysholm e, no pós-operatório, pelo IKDC. Resultados: Para melhor compreensão, os 23 indivíduos tratados foram divididos em três grupos. Vinte e um apresentaram lesão do ligamento cruzado anterior e 11 foram submetidos à reconstrução ligamentar (grupo I), com resultados considerados satisfatórios. Dez dos 21 pacientes com lesão do LCA não foram submetidos à reconstrução ligamentar (grupo II), dos quais cinco evoluíram satisfatoriamente, sem necessidade de reconstrução ligamentar, cinco, com queixa de instabilidade e foram submetidos à reconstrução ligamentar. Quatro desses pacientes apresentaram o menisco íntegro e uma ruptura do menisco medial. Dois apresentaram LCA intacto (grupo III), um evoluiu satisfatoriamente e outro apresentou ruptura do menisco lateral. De acordo com a escala de Lysholm, a média pré-operatória foi de 57,53 e a média pós-operatória, de 86,95, representando melhora estatisticamente significativa (Wilcoxon $\mathrm{p}<0,01$ ). Foi utilizada a ANOVA não paramétrica para dados ordinais com medidas repetidas para avaliar as medidas no pré e pós-operatório, considerando os grupos I e II. Avaliou-se a estabilização do joelho e não se detectou diferença estatisticamente significativa entre os grupos I e II ( $p=0,648)$. Mesmo supondo que houvesse diferença entre os dois grupos, ambos tiveram o mesmo comportamento. Na avaliação pós-operatória pelo IKDC, quatro pacientes foram classificados como A; 13, como B; e 6, como C. Dos seis pacientes com resultados $\mathrm{C}$, apenas dois apresentaram ruptura do menisco. Conclusão: Dos 23 pacientes, apenas dois apresentaram falha da cicatrização dos meniscos reparados. De acordo com a escala de Lysholm, houve melhora significativa dos pacientes tratados. A reparação do LCA não influenciou os resultados.

Descritores - Artroscopia; Reparação do menisco; Implantes

\section{ABSTRACT}

Objective: To evaluate the functional evolution of knees after repair of longitudinal meniscal rupture with absorbable arrow implant. Methods: Between June 1997 and February 2001, 23 patients with a mean age of 26.3 years were evaluated. The mean follow-up time was 72.87 months (45-96). We performed 19 medial and 4 lateral meniscal repairs. The patients were preand postoperatively evaluated regarding joint function according to the Lysholm scale, and, postoperatively, according to IKDC. Results: For better understanding, the 23 treated cases were divided into three groups. Twenty one had ACL injuries, eleven of whom were submitted to ligament reconstruction (Group I). All these 11 cases were regarded as satisfactory. The remaining 10 cases of the 21 with ACL lesion were not submitted to ligament reconstruction (Group II). Of these, 5 evolved satisfactorily, not requiring ligament reconstruction. The remaining five evolved with complaint of ligament instability, being all submitted to reconstruction. Four of these had an integral meniscus and one presented a failure of the medial meniscus. The remaining two cases who did not present ACL injury (Group III), one patient evolved satisfactorily and one developed a failure of the lateral meniscus. According to the Lysholm scale, preoperative mean score was 57.53 and the postoperative mean score was 86.95, evidencing a statistically significant improvement (Wilcoxon $p$ $<0.01$ ). The non-parametric ANOVA was employed for ordinal data with repeated measurements to assess pre- and postoperative measurements, considering Groups I and II. We assessed knee stabilization and found no statistically significant difference between Groups I and II ( $p=0.648)$. Even if there were differences between the two groups, both had the same behavior. On postoperative assessment with IKDC, 4 patients were grade A, 13 were grade $B$, and 6 were grade $C$. Two $C$ results were caused by a meniscal rupture. Conclusion: Of the 23 patients, only two presented known failures of the sutured meniscus. According to the Lysholm scale, there was a significant improvement in the treated patients. The ACL repair caused no bias on the results.

Keywords - Arthroscopy; Meniscal repair; Implants

Mestre e Doutor em Cirurgia, Universidade Estadual de Campinas-Unicamp; Chefe de Equipe de Ortopedia do Hospital São Joaquim.

Trabalho realizado pelo Serviço de Artroscopia e Ortopedia no Hospital Alemão Oswaldo Cruz

Correspondência: Al. Santos, 211, conj. 209 - 01419-002 - São Paulo, SP - Brasil. E-mail: waldo@uol.com.br 


\section{INTRODUÇÃO}

Vários autores ${ }^{(1-3)}$ analisaram o uso de implantes absorvíveis para a reparação das lesões longitudinais do menisco. Com os implantes, realiza-se essa reparação sem manipular fios e nós, evitando uma incisão complementar, o que torna o procedimento totalmente artroscópico, mais fácil e rápido. Esse é o diferencial em relação aos procedimentos sem implante. O primeiro implante disponível para uso artroscópico foi o flecha (arrow). Esse apresenta a forma de “T”, cujo eixo maior fixa a lesão do menisco e o menor o comprime (Figura 1).

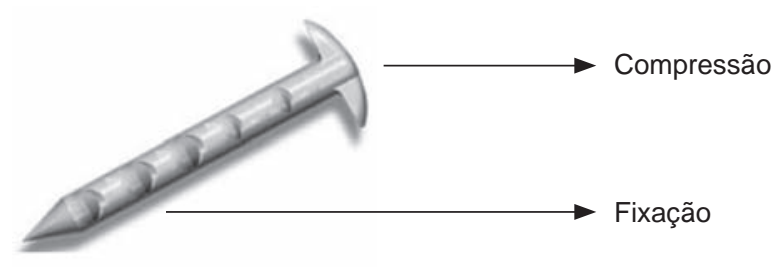

Figura 1 - Flecha

Motivados por esses benefícios, iniciamos o uso do flecha em 1997 na reparação de lesões longitudinais do menisco. A reparação ocorreu em lesões observadas durante a reconstrução do ligamento cruzado anterior (LCA), como também nas que apresentavam LCA intacto. Com base nos estudos de Steenbrugge et $\mathrm{al}^{(4)}$, Hanks et $\mathrm{al}^{(5)}$ e Koukoulias e Papastergiou ${ }^{(6)}$ os quais consideram que a instabilidade não contraindica a reparação do menisco, houve situações em que a reparação do menisco foi feita em pacientes com lesão do LCA, mas refratários a realizar a reparação ligamentar.

Reavaliamos os casos submetidos à reparação do menisco feita exclusivamente com esse tipo de implante, flecha, e desenvolvemos um estudo retrospectivo, analisando a evolução funcional dos pacientes em médio prazo. Comparamos, também, o grupo de pacientes com LCA reconstruído com o grupo que permaneceu com o LCA não tratado, para observar a influência de estabilidade nos resultados.

\section{MÉTODOS}

Revisamos os prontuários e reavaliamos todos os pacientes que apresentaram lesão vertical do menisco, localizada em área vascularizada e reparada exclusivamente com implantes absorvíveis flecha. Entre junho de 1997 e fevereiro de 2001, 26 pacientes com esse tipo de lesão foram submetidos à cirurgia e 23 foram reavaliados.

\section{Critérios de inclusão}

Lesão longitudinal do menisco, localizada entre 3mm e 5mm distal à junção menisco sinovial, em área vascularizada, com comprimento de $2 \mathrm{~cm}$ a $4 \mathrm{~cm}$. Todas as lesões eram instáveis à palpação. As lesões estáveis ou com comprimento menor do que $2 \mathrm{~cm}$ foram consideradas potencialmente cicatrizáveis e não foram tratadas. Nenhuma lesão era maior do que $60 \%$ da circunferência do menisco.

\section{Critérios de exclusão}

Foram excluídas as lesões múltiplas do menisco, com deformidades do corpo, localizadas na zona avascular, de destacamento capsulomeniscal ou aquelas cuja redução do fragmento articular necessitasse de tensão, fosse lesado ou irregular. Também foram eliminadas as lesões com reparação por flecha, mas associadas a outro tipo de fixação com fios de sutura.

\section{O implante}

O flecha foi o implante absorvível utilizado. Apresenta forma em "T", corpo com 1,1mm de diâmetro e cabeça com 4mm de comprimento. O maior eixo apresenta comprimento de $10 \mathrm{~mm}, 13 \mathrm{~mm}$ e $16 \mathrm{~mm}$; é denteado em ambos os lados, formando ângulo reto com a cabeça do implante. É composto por ácido poli-l- lático (PLA), cuja reabsorção leva de 30 a 60 meses. Após setembro de 2000, passou a ser composto por um polímero com 96\% de PLA e 4\% de ácido poli-DL-lático. Essa última composição não foi usada neste estudo.

\section{Classificação dos indivíduos}

Para melhor compreensão do estudo, dividimos os pacientes em três grupos quanto à integridade ou reparação do LCA:

Grupo I (lesão do menisco e reparação do LCA). Nesse grupo estão os pacientes com dor mecânica do joelho e que, ao exame físico, apresentavam sensibilidade na interlinha articular com sinais de Appley e McMurray, associada à queixa de instabilidade frontal da articulação, com sinal de gaveta anterior maior do que $6 \mathrm{~mm}$, comparado com o joelho contralateral avaliado por Rolimeter (Aircast). Quando submetidos à artroscopia, se fosse observada lesão longitudinal do menisco, esta seria reparada; e, se fosse confirmada a lesão do LCA, este seria reconstruído. Os pacientes estavam cientes da técnica de reconstrução do ligamento e da possibilidade de reparação do menisco.

Grupo II (lesão do menisco em joelho instável). Esses pacientes demonstravam sinais de lesão do menisco, sem queixa de instabilidade, e apresentavam gaveta anterior com o uso do Rolimeter (Aircast), menor de 6mm, 
comparada com a contralateral. Foram alertados sobre a instabilidade e possível necessidade de reconstrução ligamentar. Certos pacientes, por diversos motivos, não aceitaram a reconstrução ligamentar, mas somente a reparação do menisco. Durante a artroscopia, se fosse observada lesão do LCA associada à lesão longitudinal do menisco, este seria reparado sem procedimento na lesão ligamentar, tornando esses indivíduos o foco do estudo.

Grupo III (lesão do menisco em joelho estável). Pacientes com dor mecânica e sensibilidade na interlinha articular com sinais de Appley e McMurray presentes e com joelho estável. Pacientes submetidos à artroscopia, mas com lesão longitudinal do menisco, seriam tratados com flecha. Todos os pacientes estavam cientes da possibilidade da reparação.

\section{TÉCNICAS CIRÚRGICAS}

Os pacientes foram submetidos à artroscopia do joelho com uso de garrote pneumático e leg holder. Foram usados os portais ântero-lateral e ântero-medial. Todos foram submetidos à avaliação artroscópica, observando-se integridade ou lesão dos meniscos, cartilagem e ligamentos. A lesão do menisco, uma vez constatada, era preparada por raspagem de suas bordas com shaver. O sangramento foi estimulado por perfurações da porção parietal do menisco até o tecido sinovial perimeniscal por meio de agulha de peridural. A redução da lesão foi realizada pela aproximação das bordas, sem necessidade de tração. A dimensão transversal do provável ponto de penetração do implante na porção articular até a junção meniscocapsular da borda parietal do menisco foi medida, determinando as dimensões do flecha. A lesão foi fixada por intermédio de guia canulado e implantes aplicados. O número de implantes variou conforme a impressão visual da estabilização.

Os pacientes que apresentavam lesão sintomática do LCA, dispostos a fazer a reconstrução ligamentar, foram submetidos ao procedimento com tendão patelar ou semitendinoso e gracilis, conforme planejamento pré-operatório.

Pacientes com lesão do LCA parcial ou total, que não haviam concordado com a reconstrução ligamentar, mas apresentavam esse tipo de lesão do menisco, tiveram-na reparada sem procedimento no ligamento.

Os pacientes não foram imobilizados, permitindo-se a movimentação ativa entre $0^{\circ}$ e $90^{\circ}$. A marcha foi liberada com muletas sem carga. Após quatro dias de cirurgia, os exercícios isométricos para quadríceps foram iniciados, conforme a sensação de dor de cada indivíduo. Após 40 dias iniciou-se o tratamento por fisioterapia, com liberação progressiva da carga durante a marcha.

\section{Critérios de avaliação}

Todos foram avaliados no pré e no pós-operatório pela escala de Lysholm $^{(7)}$ e os resultados estatisticamente comparados pelo teste de Wilcoxon. Para a comparação entre os grupos (I e II) e de tempo (pré e pós-operatório), foi realizada a ANOVA não paramétrica com medidas ordinais repetida.

Todos foram avaliados no pós-operatório pelo $(\mathrm{IKDC})^{(8)}$.

Os sintomáticos com queixa de persistência de dor, edema e instabilidade foram submetidos à avaliação por ressonância magnética (RM).

Os pacientes que necessitaram de novo procedimento artroscópico foram submetidos à reavaliação articular, considerando a integridade do menisco e as possíveis lesões cartilaginosas.

Avaliamos que os pacientes assintomáticos deveriam apresentar algum grau de cicatrização.

\section{RESULTADOS}

Dos 23 pacientes avaliados, 20 eram do sexo masculino, dos quais 16 com joelho direito acometido. A idade média dos pacientes foi de 26 anos (12-40). O intervalo entre a lesão e o procedimento cirúrgico foi de aproximadamente 15 meses (1-72) e o seguimento médio, de 73 meses (45-96). Na avaliação artroscópica diagnóstica desses pacientes, 18 apresentaram cartilagem normal e em cinco havia fibrilação femoropatelar e femorotibial, mas sem exposição óssea.

Realizamos reparação em 19 meniscos mediais e quatro laterais. Nos procedimentos de menisco medial foram usados, em média, 4,17 implantes (dois a oito) e, nos de menisco lateral, média de quatro implantes (três a cinco).

Onze pacientes apresentaram sinal da gaveta anterior com deslocamento maior que $5 \mathrm{~mm}$ no pré-operatório e foram submetidos à reconstrução ligamentar conforme planejado, sendo considerados do grupo I (lesão do menisco e reparação do LCA).

Dez pacientes apresentaram gaveta anterior menor que $6 \mathrm{~mm}$, sem queixa de instabilidade, e a lesão do LCA foi constatada no exame artroscópico. Foi feita a reparação do menisco, sem reconstrução ligamentar, criandose o grupo II (lesão do menisco em joelho instável).

Dois pacientes apresentaram a lesão do menisco, mas com LCA íntegro; foram classificados como grupo III (lesão do menisco em joelho estável).

Dos 23 pacientes tratados, 21 apresentaram lesão do LCA. Houve melhora clínica em todos os pacientes do grupo I (lesão do menisco e reparação do LCA). 
Nos dez pacientes do grupo II (lesão do menisco em joelho instável) com lesão não tratada do LCA, cinco evoluíram de forma assintomática e não solicitaram a reconstrução ligamentar. Os outros cinco evoluíram com queixa importante de instabilidade e em todos foi realizada a reconstrução ligamentar; no exame artroscópico, quatro meniscos tratados estavam íntegros, confirmando os achados da RM. Um paciente apresentou ruptura do menisco medial e foi submetido a meniscectomia parcial. Nos cinco pacientes que necessitaram de nova intervenção, consideramos apenas a última graduação pelas escalas de Lysholm e IKDC antes do novo procedimento. Esses pacientes foram excluídos do estudo no pós-operatório e não foram considerados como grupo I.

Os dois casos do grupo III (lesão do menisco em joelho estável) que não apresentaram lesão do LCA no início evoluíram satisfatoriamente, mas um paciente apresentou ruptura do menisco lateral após 56 meses de seguimento (Figura 2).

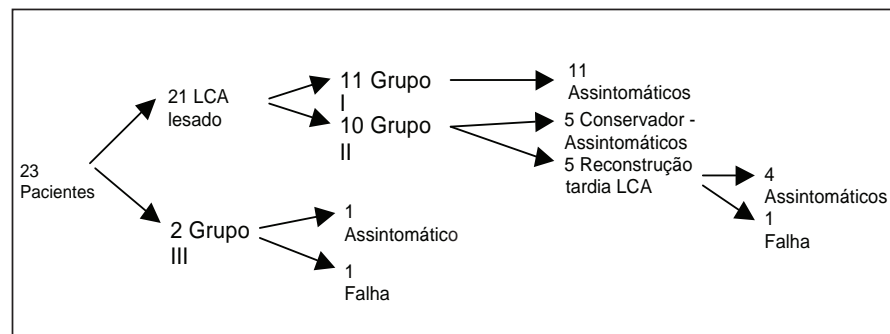

Figura 2 - Estudo dos pacientes submetidos à reparação da ruptura longitudinal do menisco pelo flecha. Grupo I: pacientes com lesão do menisco e reconstrução do LCA. Grupo II: pacientes com lesão do menisco em joelho instável. Grupo III: pacientes com lesão do menisco em joelho estável.

Dos 23 operados, apenas dois apresentaram falha na reparação, correspondendo a 8,7\% dos casos (Figura 1). Essa falha ocorreu em um paciente do grupo II e em um do grupo III.

\section{Escala de Lysholm}

Por essa avaliação, a média pré-operatória foi de 55,43 pontos (36-74) e o desvio padrão, de $\pm 13,71$; 15 pacientes apresentaram resultados fracos e oito, razoáveis. No pós-operatório, a média foi de 86,3 pontos (6-100) e o desvio padrão, de $\pm 12,7$, nove apresentando resultados excelentes; sete, obtendo bons resultados; cinco, razoáveis; e dois, fracos. Comparando o pré com o pós-operatório, houve melhora estatisticamente significativa (Wilcoxon, p < 0,01) (Tabela 1 e Gráfico 1).

Foi utilizada a ANOVA não paramétrica para dados ordinais com medidas repetidas para avaliar as medidas

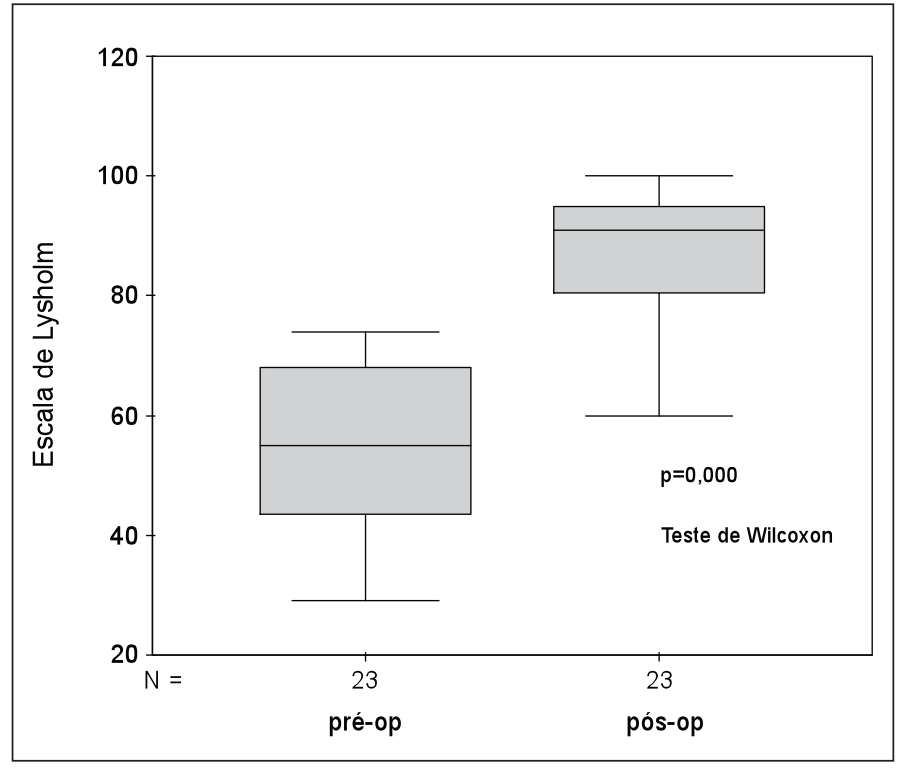

Gráfico 1 - Estudo comparativo de todos os pacientes do pré e pós-operatório pela escala de Lysholm

Tabela 1 - Avaliação no pré e pós-operatório pela escala de Lysholm

\begin{tabular}{|c|c|c|c|}
\hline Indivíduos & Pré-operatório & Pós-operatório & LCA \\
\hline I & 68 & 86 & Grupo I \\
\hline II & 55 & 71 & Grupo I \\
\hline III & 44 & 85 & Grupo II \\
\hline IV & 55 & 60 & Grupo I \\
\hline V & 68 & 85 & Grupo II \\
\hline VI & 55 & 100 & Grupo I \\
\hline VII & 36 & 91 & Grupo II \\
\hline VIII & 68 & 95 & Grupo I \\
\hline IX & 55 & 86 & Grupo II \\
\hline$x$ & 43 & 91 & Grupo I \\
\hline $\mathrm{XI}$ & 59 & 95 & Grupo I \\
\hline XII & 59 & 100 & Grupo I \\
\hline XIII & 74 & 95 & Grupo I \\
\hline XIV & 68 & 81 & Grupo II \\
\hline$x V$ & 74 & 95 & Grupo II \\
\hline $\mathrm{XVI}$ & 51 & 68 & Grupo II \\
\hline $\mathrm{XVII}$ & 42 & 100 & Grupo II \\
\hline$X V I I I$ & 38 & 100 & Grupo II \\
\hline $\mathrm{XIX}$ & 65 & 91 & Grupo I \\
\hline$x X$ & 74 & 64 & Grupo II \\
\hline$X X I$ & 61 & 66 & Grupo II \\
\hline XXII & 29 & 80 & Grupo I \\
\hline XXIII & 34 & 100 & Grupo II \\
\hline MÉDIA & 55,43 & 86,3 & \\
\hline
\end{tabular}

Indivíduos = Pacientes operados. Pré-operatório = Resultado pela escala de Lysholm de cada indivíduo no pré-operatório. Pós-operatório = Resultado pela escala de Lysholm de cada indivíduo no pós-operatório. Estado do LCA encontrado no pós-operatório imediato. Grupo I: pacientes com no pós-operatório. Estado do LCA encontrado no pós-operatório imediato. Grupo I: pacientes com
lesão do menisco e reconstrução do LCA. Grupo II: pacientes com lesão do menisco em joelho instável. Grupo III: pacientes com lesão do menisco em joelho estável. Média: média aritmética entre o pré e o pós-operatório. Fonte: Hospital São Joaquim 
no pré e pós-operatório, considerando os grupos I e II. Essa abordagem permite testar hipóteses de inexistência do efeito de fatores entre indivíduos (grupos I e II) e intraindivíduos (pré e pós-operatório) e a interação desses fatores (Tabela 2).

Tabela 2 - Avaliação no pré e pós-operatório pela escala de Lysholm nos pacientes submetidos à reconstrução do LCA e com LCA lesado

\begin{tabular}{l|c|c|c}
\hline & Estatística do teste & g.l. & p-valor \\
\hline Entre indivíduos (grupo) & 0,209 & 1 & 0,648 \\
\hline Intraindivíduos (tempo) & 45,532 & 1 & $<0001$ \\
\hline Interação & 0,034 & 1 & 0,854 \\
\hline
\end{tabular}

Estatística do teste: ANOVA não paramétrica para dados ordinais com medidas repetidas. Pvalor: valor do p. Entre indivíduos (grupo): Comparação do grupo I e do grupo II. Intraindivíduos (tempo): Comparação entre pré-operatório e pós-operatório.

Fonte: Hospital São Joaquim

Avaliamos a estabilização do joelho e não detectamos diferença estatisticamente significativa entre os grupos I e II ( $\mathrm{p}=0,648)$. Mesmo supondo que houvesse diferença entre os dois grupos, ambos tiveram o mesmo comportamento (Gráfico 2). Não foi encontrado efeito de interação, mas houve diferença entre o período préoperatório e o pós-operatório.

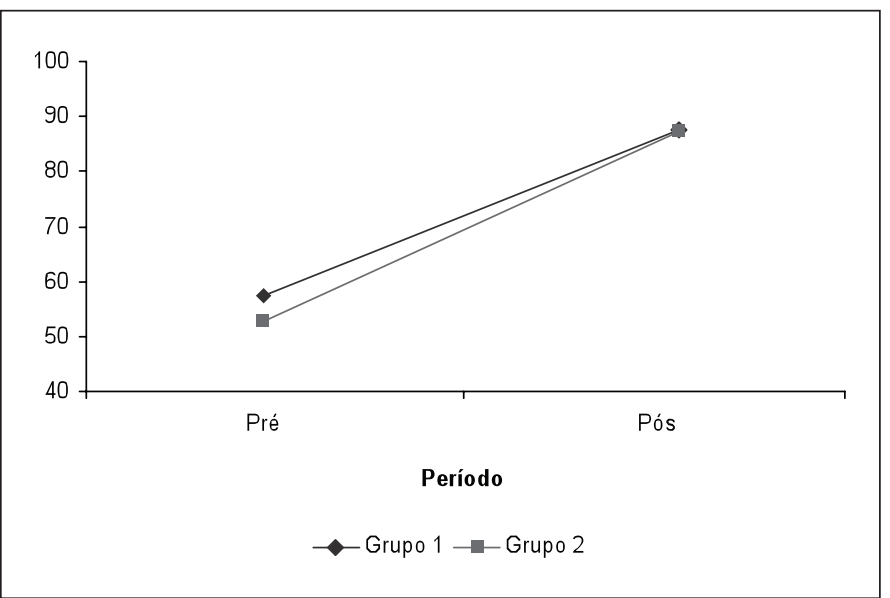

Gráfico 2 - Estudo comparativo dos pacientes dos grupos I e II no pré e pós-operatório pela escala de Lysholm

Gráfico da ANOVA não paramétrica para dados ordinais com medidas repetidas. P-valor: valor do p. Abscissa: valor da escala de Lysholm. Ordenada: tempo no pré-operatório e pós-operatório. Intraindivíduos: comparação do grupo I e do grupo II.

A avaliação pós-operatória desses pacientes demonstrou que quatro queixavam-se de claudicação, sendo três do grupo II (lesão do menisco em joelho instável) e dois do grupo III (lesão do menisco em joelho estável). A claudicação estava relacionada com a instabilidade em dois pacientes, mas nos outros dois houve falha na reparação do menisco.
Todos os pacientes mostraram-se capazes de andar sem muletas ou bengalas.

Seis queixaram-se de certo grau de rigidez articular, sendo quatro do grupo I (lesão do menisco e reparação do LCA), dois do grupo II (lesão do menisco em joelho instável).

Considerando o item estabilidade, observou-se que três pertenciam ao grupo I (lesão do menisco e reparação do LCA); seis, ao grupo II (lesão do menisco em joelho instável) e um, ao grupo III (lesão do menisco em joelho estável).

Treze pacientes queixaram-se de dor, a qual se apresentou mais importante em um do grupo III (lesão do menisco em joelho estável) com ruptura do menisco. Doze pacientes referiam dor ao esforço, sendo quatro do grupo I (lesão do menisco e reparação do LCA) e oito do grupo II (lesão do menisco em joelho instável); um paciente do último grupo apresentou nova lesão.

Quatro pacientes referiam edema, sendo dois do grupo I (lesão do menisco e reparação do LCA) e dois do grupo II (lesão do menisco em joelho instável).

Nove relataram pequena restrição para subir escadas, sendo quatro do grupo I (lesão do menisco e reparação do LCA), três do grupo II (lesão do menisco em joelho instável) e dois do grupo III (lesão do menisco em joelho estável).

\section{IKDC}

A avaliação pelo IKDC no pós-operatório encontrou quatro pacientes com classificação A; 13, com B; seis, com C. Desses, 12 afirmaram não haver mudanças relacionadas com a atividade, consequentemente, com a lesão do joelho. Dois pacientes foram classificados como B no exame radiológico, mas nenhum apresentou evolução para artrose, conforme os critérios radiológicos (Tabela 3).

Foram considerados normais: um paciente do grupo I (lesão do menisco e reparação do LCA) e quatro do grupo II (lesão do menisco em joelho instável). Consideraram-se joelhos quase normais: oito pacientes do grupo I (lesão do menisco e reparação do LCA), três do grupo II (lesão do menisco em joelho instável) e um do grupo III (lesão do menisco em joelho estável).

Consideraram-se joelhos anormais: dois pacientes do grupo I (lesão do menisco e reparação do LCA), três do grupo II (lesão do menisco em joelho instável) e um do grupo III (lesão do menisco em joelho estável).

Reavaliamos os seis pacientes classificados como C. Dois apresentaram lesão do menisco. Um paciente do 
Tabela 3 - Resultado do IKDC no pós-operatório

\begin{tabular}{|c|c|c|}
\hline Indivíduos & IKDC & Estado do LCA \\
\hline I & $B$ & Grupo I \\
\hline II & C & Grupo I \\
\hline III & $\mathrm{A}$ & Grupo II \\
\hline IV & C & Grupo I \\
\hline V & B & Grupo II \\
\hline $\mathrm{VI}$ & $B$ & Grupo I \\
\hline VII & B & Grupo II \\
\hline VIII & B & Grupo I \\
\hline IX & C & Grupo II \\
\hline$x$ & B & Grupo I \\
\hline$X I$ & $B$ & Grupo I \\
\hline XII & B & Grupo I \\
\hline XIII & $\mathrm{B}$ & Grupo I \\
\hline XIV & $B$ & Grupo III \\
\hline$x V$ & B & Grupo II \\
\hline $\mathrm{XVI}$ & C & Grupo III \\
\hline $\mathrm{XVII}$ & A & Grupo II \\
\hline $\mathrm{XVIII}$ & A & Grupo II \\
\hline $\mathrm{XIX}$ & $B$ & Grupo I \\
\hline$X X$ & C & Grupo II \\
\hline$X X I$ & C & Grupo II \\
\hline XXII & $A$ & Grupo I \\
\hline XXIIII & A & Grupo II \\
\hline
\end{tabular}

Indivíduo refere-se a cada paciente. IKDC resultado A - Normal, B - Quase normal, C - Anormal, D - Severamente anormal. LCA - Estado do LCA (ligamento cruzado anterior). Grupo I: pacientes com lesão do menisco e reconstrução do LCA. Grupo II: pacientes com lesão do menisco em joelho instável. Grupo III: pacientes com lesão do menisco em joelho estável. Média: media aritmética entre o pré e o pós-operatório.

Fonte: Hospital São Joaquim

grupo III (lesão do menisco em joelho estável) apresentou lesão do lateral e um paciente do grupo II (lesão do menisco em joelho instável), lesão do medial.

Dois pacientes do grupo II (lesão do menisco em joelho instável) evoluíram com instabilidade importante, contudo, a reparação do menisco permaneceu intacta, o que foi confirmado pela avaliação artroscópica.

Dois pacientes do grupo I (lesão do menisco e reparação do LCA) foram classificados como C. Um referiu dor à compressão da cicatriz medial e paresia da face medial da perna. Foi submetido a RM e a reparação do menisco pareceu intacta; esse paciente não referiu dor mecânica. Outro paciente apresentou dor anterior do joelho à compressão da patela quando ajoelhado, porém, sem dor mecânica; o controle por RM mostrou menisco intacto; a dor estaria relacionada com a fratura da patela, ocorrida durante o procedimento cirúrgico.

Dos oito pacientes com queixa do joelho que foram avaliados por RM, apenas dois apresentaram lesão do menisco.
Não se observaram complicações neurovasculares, sinais de sinovite, granuloma subcutâneo, granuloma de corpo estranho. Nos pacientes reavaliados artroscopicamente, não foram observadas lesões condriais nem fragmentos do implante intra-articular. Contudo, algumas dessas complicações podem não ter sido detectadas neste estudo, principalmente no grupo assintomático, porque não houve controle artroscópico ou por RM.

\section{DISCUSSÃO}

Os resultados deste estudo indicam que houve melhora funcional dos pacientes submetidos à reparação do menisco com flecha e apenas duas falhas de consolidação conhecidas.

Na escolha de implante absorvível, é necessário considerar a estabilização dos fragmentos e se o conjunto menisco reparado e o implante podem resistir às mesmas cargas suportadas pelas suturas com fios. Estudos comparativos indicam que a resistência mecânica inicial da sutura do menisco com fios é superior à dos implantes. De acordo com Song e Lee ${ }^{(9)}$, a sutura vertical com PDS apresenta resistência de $114 \mathrm{~N}$ contra 38N do flecha, mas ressaltaram que quando dois flechas são aplicados à distância de $6 \mathrm{~mm}$, essa resistência sobe para $57 \mathrm{~N}$.

Barber e Herbert ${ }^{(10)}$, em seu estudo, observaram que a resistência do Mersilene 2.0 é de $113 \mathrm{~N}$ e a do flecha, 34N. Becker et $\mathrm{al}^{(11)}$ encontraram resistência do Ethibond de 62,0N contra o flecha, com 24,7N. Esses estudos mecânicos foram realizados em animais e cadáveres e indicam resistência maior dos fios no momento da aplicação em determinada área do menisco, mas não consideram todo o conjunto do menisco reparado, nem a resistência durante e após o período de cicatrização. Arnoczky e Lavagnino ${ }^{(12)}$ compararam a sutura com o fio PDS com implantes em PLA e observaram que inicialmente há resistência superior com o fio; contudo, após seis semanas, o implante manteve a sua tensão e o fio apresentou sinas de afrouxamento. A fixação dos fragmentos fornecida pelo flecha foi suficiente para permitir melhora clínica em nosso estudo. Nos oito pacientes sintomáticos submetidos à RM, dois apresentaram lesão do menisco, mas apenas os cinco em que houve reconstrução do LCA em um segundo tempo foli realizada nova avaliação artroscópica da integridade do menisco.

O uso do flecha possibilitou reparação do menisco por via exclusivamente artroscópica, sem uso de um nó de fixação, o que nos pareceu uma evolução importante. Antes dos implantes absorvíveis, a reparação do menisco por via artroscópica era realizada exclusivamente 
com diferentes tipos de fios e a passagem da sutura feita de dentro da articulação para fora e de fora para dentro. A reparação do menisco de dentro para fora necessita de incisão complementar até a cápsula na face pósteromedial ou lateral do joelho, para proteger as estruturas neurovasculares na passagem das agulhas com os fios; a fixação do nó é extra-articular. Cannon e Vittori ${ }^{(13)}$ avaliaram esse procedimento, associando a reconstrução do LCA quando necessária e encontraram 82\% pacientes assintomáticos. Nos casos em que não havia lesão do LCA, apenas 48\% permaneceram assintomáticos. Rubman et $\mathrm{al}^{(14)}$ realizaram sutura de menisco nas áreas vascularizadas com seguimento de 42 meses e obtiveram $80 \%$ de resultados favoráveis.

Outra técnica cirúrgica para a reparação do menisco é feita pela passagem do fio de sutura de fora para dentro e destaca-se por alcançar todas as suas áreas, em particular o corno anterior. Morgan et $\mathrm{al}^{(15)}$ avaliaram-na pela aplicação do nó extra-articular e encontraram 96\% de casos assintomáticos e 3\% de falhas. Reavaliando o grupo assintomático, observaram 84\% de cicatrização (65\% de cicatrização completa e 19\%, incompleta). Reigel et $\mathrm{al}^{(16)}$ também avaliaram a reparação do menisco por sutura totalmente artroscópica e o nó totalmente intra-articular, observando que todos permaneceram assintomáticos. Abdelkafy et $\mathrm{al}^{(17)}$, usando essa técnica, reavaliaram 52 dos 93 pacientes operados por esse procedimento e, em seguimento de 12 anos, encontraram Lysholm de 87,29; 36 apresentaram IKDC classificado como A, concluindo que é um procedimento seguro e de bons resultados. Esse processo apresenta resultados satisfatórios, mas também usa uma incisão complementar, expondo as estruturas vasculonervosas a riscos, além de necessitar de vários nós intra ou extra-articulares, o que não ocorre com os implantes.

Certos estudos comparam o flecha às suturas. AlbrechtOlsen et $\mathrm{al}^{(1)}$ compararam a sutura horizontal com o flecha e avaliaram a reparação por via artroscópica após três a quatro meses. Observaram a cicatrização em 91\% dos casos por flecha e $75 \%$, por sutura. Steenbrugge et $\mathrm{al}^{(4)} \mathrm{e}$ Hürel et $\mathrm{al}^{(2)}$ compararam a sutura de dentro para fora com o flecha, percebendo que o uso do implante proporcionava procedimento com menor tempo cirúrgico, o que é tecnicamente mais fácil, com menor risco vasculonervoso, principalmente ao se manipular o corno posterior.

Existem controvérsias quanto ao uso do flecha. Petsche et $\mathrm{al}^{(3)}$ avaliaram pacientes submetidos a meniscorrafia com flecha e observaram melhora estatisticamente significativa nas escalas Lysholm e Tegner. Houve dois casos de nova ruptura em paciente que apresentava lesão do LCA; o primeiro em um com o ligamento não tratado e outro naquele submetido à reconstrução do LCA. Por outro lado, Kurzweil et al ${ }^{(19)}$ encontraram falhas de $20 \%$ em meniscorrafias por flecha, associadas à ligamentoplastia, e, nas lesões isoladas do menisco, 42\%, considerando insatisfatório esse tipo de fixação.

Nosso estudo apresenta limitações por ser retrospectivo, com amostra limitada, sem reavaliação artroscópica nos pacientes tratados. A certeza da cicatrização ou lesão do menisco só foi possível em seis pacientes submetidos à reavaliação artroscópica. Por critérios clínicos, consideramos que os pacientes com resultados funcionais satisfatórios apresentaram algum grau de cicatrização do menisco; contudo, acreditamos que existiu falha não detectada na cicatrização do menisco, uma vez que esses indivíduos não foram reavaliados por RM ou por nova artroscopia diagnóstica.

Talvez, algumas lesões que tratamos cicatrizassem sem nenhum tipo de fixação, particularmente no grupo em que foi feita a reconstrução do LCA. Weiss et $\mathrm{al}^{(20)}$ consideraram que lesões longitudinais que tendem a ocorrer na porção vascularizada da periferia do menisco possuem grande potencial de cura e não devem ser tratadas, salvo alguma anormalidade. Por outro lado, Talley e Grana ${ }^{(21)}$ acreditam que as lesões longitudinais estáveis do menisco apresentam grande potencial de não cicatrização provocada pela propagação da lesão; portanto, deveriam ser reparadas. Pierre et $\mathrm{al}^{(22)}$ estudaram as lesões do menisco não tratadas em pacientes submetidos à reconstrução do LCA e concluíram que as lesões do menisco medial superiores a $10 \mathrm{~mm}$ devem ser tratadas. Yagishita et al ${ }^{(23)}$ observaram que, não obstante o potencial de cicatrização dessas lesões do menisco encontradas durante a reconstrução do LCA, as lesões mais longas devem ser reparadas. Em nosso estudo, todas as lesões viáveis acima de $20 \mathrm{~mm}$ foram reparadas e as menores, excluídas da análise.

A necessidade de reparação imediata do LCA em casos de reparação do menisco é um assunto controverso. Steenbrugge et $\mathrm{al}^{(4)}$ compararam pacientes submetidos à reparação do menisco com reparação do LCA com os do grupo de joelhos instáveis. Dos 20 pacientes tratados, 14 apresentaram resultado satisfatório; por outro lado, sete foram submetidos à reconstrução em segundo tempo. Concluíram que, mesmo que os resultados insatisfatórios sejam maiores nos pacientes com joelhos instáveis, a lesão do LCA não contraindica a reparação do menisco. Hanks et al $^{(5)}$ realizaram 23 
reparações do menisco em joelhos instáveis, sendo 12 por artrotomia e 11 por artroscopia, e observaram nova ruptura em apenas três casos. Desses pacientes, seis referiam dor, mas só um necessitou de analgésico. Oito pacientes referiam instabilidade e um foi submetido à reconstrução do LCA. Também consideraram que a instabilidade não contraindica a reparação do menisco. Mais recentemente, Koukoulias e Papastergiou ${ }^{(6)}$ avaliaram 11 reparações do menisco medial em pacientes com joelho instável por lesão do LCA com flecha. Encontraram falha em três indivíduos. Oito permaneceram assintomáticos nas atividades diárias, mas sete queixavam-se de edema após esporte. Concluíram que a deficiência do LCA não contraindica a reparação do menisco, mas há a necessidade de diminuir o nível do esporte. Em nosso estudo, a reparação ou lesão do ligamento cruzado anterior não influíram nos resultados, considerando a escala de Lysholm. Foi relevante que, em nosso estudo, 10 pacientes com lesão do LCA não foram submetidos à reconstrução ligamentar imediata e, apesar de instabilidade articular, cinco permaneceram assintomáticos e houve certo grau de cicatrização do menisco. Outros cinco pacientes apresentaram queixa de instabilidade, em menor grau de dor e, na revisão cirúrgica para reconstrução ligamentar, em apenas um paciente foi observada a ruptura do menisco. Mesmo assim, todos apresentaram alguma melhora funcional.

\section{REFERÊNCIAS}

1. Albrecht-OlsenP, Kristensen G, Burgaard P, Joergensen $U$, Toerholm C. The arrow versus horizontal suture in arthroscopic meniscus repair. A prospective randomized study with arthroscopic evaluation. Knee Surg Sports Traumatol Arthrosc. 1999;7(5):268-73

2. Hürel C, Mertens F, Verdonk R. Biofix resorbable meniscus arrow for meniscal ruptures: results of a 1-year follow-up. Knee Surg Sports Traumatol Arthrosc. 2000;8(1):46-52.

3. PetscheTS, Selesnick H, Rochman A. Arthroscopic meniscus repair with bioabsorbable arrows.Arthroscopy. 2002;18(3):246-53.

4. SteenbruggeF, Verdonk R, Hurel C, Verstraete K. Arthroscopic meniscus repair: inside-out technique vs. Biofix meniscus arrow. Knee Surg Sports Traumatol Arthrosc. 2004;12(1):43-9.

5. Hanks G, Gause TM, Handal JA, Kalenak A.Meniscus repair in the anterior cruciate deficient knee. Am J Sports Med. 1990;18:606-13.

6. Koukoulias N, Papastergiou S. Mid-term clinical results of medial meniscus repair with the meniscus arrow in the unstable knee. Knee Surgery, Sports Traumatology, Arthroscopy. 2006;15(2):138-43.

7. Tegner $Y$, Lysholm J. Rating systems in the evaluation of knee ligament injuries. Clin Orthop. 1985;198:43-9.

8. HeftiF, Muller W, Jakob RP, Staubli HU. Evaluation of knee ligament injuries with the IKDC form. Knee Surg Sports Traumatol Arthrosc. 1993;1:226-34.

9. Song EK, Lee KB. Biomechanical test comparing the load to failure of the biodegradable meniscus arrow versus meniscal suture. Arthroscopy. 1999;15(7):726-32.

10. Barber FA, Herbert MA. Meniscal repair devices. Arthroscopy. 2000;16(6):613-8.

11. BeckerR, Wirz D, Wolf C, Gopfert B, Nebelung W, Friederich N. Measurement of meniscofemoral contact pressure after repair of bucket-handle tears with biodegradable implants. Arch Orthop Trauma Surg. 2005;125(4):254-60.

12. ArnoczkySP, Lavagnino M. Tensile fixation strengths of absorbable meniscal repair devices as a function of hydrolysis time. An in vitro experimental study. Am J Sports Med. 2001;29(2):118-23.
No grupo III (lesão do menisco em joelhos estáveis) não havia pacientes suficientes para comparação estatística com os outros grupos. Dois indivíduos apresentaram LCA intacto; um permaneceu assintomático e o outro sofreu nova entorse após quatro anos com ruptura do menisco lateral (IKDC $=6$ e Lysholm $=68$ ). Foi o grupo menos expressivo, mas seu resultado poderia ter sido influenciado pela qualidade do menisco. Cannon e Vittori $^{(13)}$ observaram que as lesões do corno posterior do menisco em joelhos sem lesão do LCA apresentam alterações degenerativas, o que comprometeria nosso critério de integridade do menisco. Por outro lado, Sommerlath $^{(24)}$ observou que, não obstante a incidência importante de ruptura nos meniscos reparados em joelhos estáveis, os que cicatrizam apresentam melhor função e menor evolução para artrose, se comparados com os submetidos à meniscectomia imediata. Portanto, reparamos os meniscos nessa situação.

\section{CONCLUSÃO}

Houve melhora da função articular desses pacientes. Dos 23 operados, apenas dois apresentaram uma falha conhecida na reparação do menisco. A lesão do LCA não influiu nesse grupo quanto ao resultado funcional de reparação. Houve maior incidência de nova intervenção nos pacientes com lesão do LCA.

13. CannonWD Jr, Vittori JM. The incidence of healing in arthroscopic meniscal repairs in anterior cruciate ligament-reconstructed knees versus stable knees. Am J Sports Med. 1992;20(2):176-81.

14. Rubman MH, Noyes FR, Barber-Westin SD. Arthroscopic repair of meniscal tears that extend into the avascular zone. A review of 198 single and complex tears. Am J Sports Med. 1998;26(1):87-95.

15. MorganCD, Wojtys EM, Casscells CD, Casscells SW. Arthroscopic meniscal repair evaluated by second-look arthroscopy. Am J Sports Med. 1991;19(6):632-7.

16. ReigelCA, Mulhollan JS, Morgan CD. Arthroscopic all-inside meniscus repair. Clin Sports Med. 1996;15(3):483-98.

17. Abdelkafy A, Aigner N, Zada M, Elghoul Y, Abdelsadek $\mathrm{H}$, Landsiedl $\mathrm{F}$. Two to nineteen years follow-up of arthroscopic meniscal repair using the outside-in technique: a retrospective study. Arch Orthop Trauma Surg. 2007;127(4):245-52.

19. KurzweilPR, Tifford CD, Ignacio EM. Unsatisfactory clinical results of meniscal repair using the meniscus arrow. Arthroscopy. 2005;21(8):905.

20. Weiss CB, Lundberg M, Hamberg P, DeHaven KE, Gillquist J. Non-operative treatment of meniscal tears. J Bone Joint Surg Am. 1989;71(6):811-22.

21. Talley MC, Grana WA. Treatment of partial meniscal tears identified during anterior cruciate ligament reconstruction with limited synovial. Arthroscopy. 2000;16(1):6-10.

22. PierreA, Hulet C, Locker B, Schiltz D, Delbarre JC, Vielpeau C. Outcome of 95 stable meniscal tears left in place after reconstruction of the anterior cruciate ligament. Rev Chir Orthop Reparatrice Appar Mot. 2001;87(7):661-8.

23. YagishitaK, Muneta T, Ogiuchi T, Sekiya I, Shinomiya K. Healing potential of meniscal tears without repair in knees with anterior cruciate ligament reconstruction. Am J Sports Med. 2004;32(8):1953-61.

24. SommerlathKG. Results of meniscal repair and partial meniscectomy in stable knees. Int Orthop. 1991;15(4):347-50. 